\title{
QED and QCD helicity amplitudes in parton-shower gauge
}

\author{
Kaoru Hagiwara ${ }^{1,2, a}$, Junichi Kanzaki ${ }^{3,4, b}$, Kentarou Mawatari ${ }^{5, \mathrm{c}}$ \\ ${ }^{1}$ Institute of Science and Engineering, Shimane University, Matsue 690-8504, Japan \\ ${ }^{2}$ KEK Theory Center, Tsukuba 305-0801, Japan \\ ${ }^{3}$ KEK and Sokendai, Tsukuba 305-0801, Japan \\ ${ }^{4}$ Present address: Kavli Institute for the Physics and Mathematics of the Universe, The University of Tokyo, Kashiwa 277-8583, Japan \\ ${ }^{5}$ Faculty of Education, Iwate University, Morioka, Iwate 020-8550, Japan
}

\begin{abstract}
We introduce photon and gluon propagators in which the scalar polarization component is subtracted systematically by making use of the BRST invariance of the off-shell vector boson created from physical on-shell states. The propagator has the light-cone gauge form, where the spacial component of the gauge vector points along the negative of the off-shell vector boson momentum. We call the gauge as parton-shower gauge, since in collinear configurations the absolute value squared of each Feynman amplitude reproduces all the singular behaviors of the corresponding parton shower in this gauge. We introduce new HELAS codes that can be used to calculate the tree-level helicity amplitudes of arbitrary QED and QCD processes by using MadGraph. The absence of subtle gauge cancellation among Feynman amplitudes allows numerical codes to evaluate singular behaviors accurately, and helps us gaining physical insights on interference patterns.
\end{abstract}

\section{Introduction}

HELAS (HELicity Amplitude Subroutines) is a set of Fortran subroutines which enable us to compute the helicity amplitudes of an arbitrary tree-level Feynman diagram [1,2]. The code has been used in various event generators such as MadGraph5_aMC@NLO (MG5aMC) [3]. ${ }^{1}$

HELAS employs the Feynman-gauge propagator for massless gauge bosons. It often leads to subtle gauge cancella-

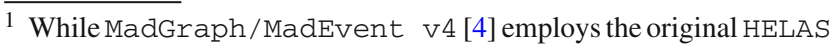
library, MG5aMC adopts ALOHA [5], which automatically generates the HELAS library by using the UFO format [6].
}

\footnotetext{
a e-mail: kaoru.hagiwara@kek.jp

b e-mail: junichi.kanzaki@ipmu.jp

c e-mail: mawatari@iwate-u.ac.jp (corresponding author)
}

tion among Feynman amplitudes, that sometimes give rise to a total loss of accuracy even with double precision. For example, the size of cancellation among amplitudes for $e^{+} e^{-} \rightarrow e^{+} e^{-} Z$ at $2 \mathrm{TeV}$ collision amounts up to $\sim 10^{12}$ and that results in about $10 \%$ inaccuracy of the calculation of the total cross section [7].

In this article, we propose a different gauge choice for the propagator for massless gauge bosons, in which the scalar polarization component is subtracted systematically by making use of the BRST invariance of the off-shell vector boson created from physical on-shell states. The propagator has the light-cone gauge form, and we may call the gauge as partonshower (PS) gauge, since, as shown below, the absolute value squared of each Feynman amplitude reproduces all the singular behaviors of the corresponding parton shower in this gauge. $^{2}$

After introducing the PS gauge in Sect. 2, we discuss the relation between the off-shell vector boson currents and the parton splitting amplitudes in Sect. 3. In Sect. 4 we give sample numerical results to demonstrate that calculations in the PS gauge can avoid subtle gauge cancellations among Feynman amplitudes by comparing with those in the Feynman gauge. Sect. 5 presents our brief summary. In Appendix A we give new HELAS subroutines in the PS gauge.

\section{Parton-shower gauge}

In this section, we introduce PS gauge for massless gaugeboson propagators.

\footnotetext{
2 After completion of the paper, we found that the same form of the gluon propagator is used indeed in a parton-shower program by Nagy and Soper [8,9].
} 
The numerator for a massless gauge boson propagator in a covariant gauge can be decomposed as

$$
\begin{aligned}
-g^{\mu \nu}+(1-\xi) \frac{q^{\mu} q^{\nu}}{q^{2}}= & \sum_{\lambda= \pm, 0} \eta_{\lambda} \epsilon^{\mu}(q, \lambda) \epsilon^{\nu}(q, \lambda)^{*} \\
& -\xi \eta_{S} \epsilon^{\mu}(q, S) \epsilon^{\nu}(q, S)^{*}
\end{aligned}
$$

where $\xi$ is a gauge parameter; $\eta_{\lambda}=\eta_{S}=1$ when $q^{2}>0$, while $\eta_{\lambda}=(-1)^{\lambda+1}$ and $\eta_{S}=-1$ when $q^{2}<0 ; \epsilon^{\mu}(q, \lambda)$ is the polarization vector with momentum $q$ and helicity $\lambda$, while $\epsilon^{\mu}(q, S)$ is the scalar polarization state

$\epsilon^{\mu}(q, S)=\frac{q^{\mu}}{\sqrt{\left|q^{2}\right|}}$.

The polarization vectors for the three helicity states satisfy

$q_{\mu} \epsilon^{\mu}(q, \lambda)=0$,

and are normalized as

$\epsilon_{\mu}(q, \lambda) \epsilon^{\mu}\left(q, \lambda^{\prime}\right)^{*}=-\delta_{\lambda \lambda^{\prime}}$.

The gauge invariance of the scattering amplitudes in QED and QCD tells that the term proportional to $q^{\mu} q^{v}$ do not affect the amplitudes, and we can express the propagator in terms of the three polarization vectors as

$-g^{\mu \nu}+\frac{q^{\mu} q^{\nu}}{q^{2}}=\sum_{\lambda= \pm, 0} \eta_{\lambda} \epsilon^{\mu}(q, \lambda) \epsilon^{\nu}(q, \lambda)^{*}$.

We note here that the longitudinally polarized vector-boson wave function can be decomposed into two parts

$\epsilon^{\mu}(q, 0)=\tilde{\epsilon}^{\mu}(q, 0)+\epsilon^{\mu}(q, S)$,

where at high energies $\left(\left|q^{2}\right| /|\mathbf{q}|^{2} \ll 1\right)$ the $\tilde{\epsilon}^{\mu}(q, 0)$ term is suppressed. The longitudinal polarization contribution to the propagator (5) hence has four components:

$$
\begin{aligned}
\epsilon^{\mu}(q, 0) \epsilon^{\nu}(q, 0)^{*}= & \tilde{\epsilon}^{\mu}(q, 0) \tilde{\epsilon}^{v}(q, 0)^{*}+\tilde{\epsilon}^{\mu}(q, 0) \epsilon^{\nu}(q, S)^{*} \\
& +\epsilon^{\mu}(q, S) \tilde{\epsilon}^{v}(q, 0)^{*}+\epsilon^{\mu}(q, S) \epsilon^{\nu}(q, S)^{*} .
\end{aligned}
$$

The last term does not contribute because it has the same form as the gauge term in (1). In fact, neither the second nor the third term contributes to the scattering amplitudes, because the BRST invariance of the quantum gauge field theory [10, 11] ensures that the gauge fixing condition annihilates the physical states:

$\left\langle\right.$ phys $\left|\partial_{\mu} A^{\mu}\right|$ phys $\rangle=0$, for the covariant gauge fixing. Because the scattering amplitudes are transitions among physical states, the scalar polarization component $\epsilon^{\mu}(q, S)$ in all the propagators of the treelevel amplitudes annihilates the physical states that gives rise to the off-shell vector boson current. In short, only the first term in the right-hand side of Eq. (7) contributes to the scattering amplitudes at the tree-level in QED and QCD.

We now introduce a tensor

$\tilde{g}^{\mu \nu}=g^{\mu \nu}-\frac{n^{\mu} q^{v}+q^{\mu} n^{v}}{n \cdot q}$

with

$n^{\mu}=\left(\operatorname{sgn}\left(q^{0}\right),-\mathbf{q} /|\mathbf{q}|\right)$,

and obtain the projected polarization vector as

$\tilde{\epsilon}^{\mu}(q, \lambda)=\tilde{g}^{\mu \nu} \epsilon_{v}(q, \lambda)$.

Here the transverse polarization states remain the same, while the longitudinal state is subtracted by the scalar state:

$\begin{aligned} \tilde{\epsilon}^{\mu}(q, \pm) & =\epsilon^{\mu}(q, \pm), \\ \tilde{\epsilon}^{\mu}(q, 0) & =\epsilon^{\mu}(q, 0)-\epsilon^{\mu}(q, S),\end{aligned}$

for all $q^{\mu}$. The BRST invariance (8) tells that only the projected polarization vectors contribute, and the propagator can be expressed as

$$
\begin{aligned}
\sum_{\lambda= \pm, 0} \eta_{\lambda} \tilde{\epsilon}^{\mu}(q, \lambda) \tilde{\epsilon}^{\nu}(q, \lambda)^{*} & =\tilde{g}^{\mu \alpha} \tilde{g}^{\nu \beta}\left(-g_{\alpha \beta}+\frac{q_{\alpha} q_{\beta}}{q^{2}}\right) \\
& =-g^{\mu \nu}+\frac{n^{\mu} q^{\nu}+q^{\mu} n^{\nu}}{n \cdot q} \\
& =-\tilde{g}^{\mu \nu} .
\end{aligned}
$$

It has the form of the light-cone gauge propagator, ${ }^{3}$ but we should note that the gauge vector (10) depends on the three momentum of the virtual vector boson. We call the propagator form (14) as the "parton-shower (PS)" gauge because of the property of the splitting amplitudes calculated in this method, as shown in the next section.

\footnotetext{
3 The light-cone gauge propagator with the gauge vector (14) was introduced in Ref. [12] in order to obtain splitting amplitudes for massive vector bosons in the standard model. In spontaneously broken gauge theories, the BRST invariance (8) takes the form $\langle$ phys $|\left(\partial_{\mu} V^{\mu}+\right.$ $\left.\xi m_{V} \chi_{V}\right) \mid$ phys $\rangle=0$, relating the scalar polarization of the massive vector boson and the corresponding Goldstone boson contributions. The gauge is named Goldstone-equivalence gauge because the dominant piece of the longitudinal polarization contribution is replaced by the Goldstone boson exchange amplitudes. One can obtain numerical codes that replaces the unitary gauge propagators by the sum of the light-cone gauge propagator of the vector boson and the Goldstone boson [13].
} 


\section{Relation to the splitting amplitudes}

In this section, we discuss the relation between the off-shell vector boson currents and the parton splitting amplitudes.

We consider an amplitude for processes with initial state radiations (space-like; $q^{2}<0$ ) that produce a nearly on-shell virtual gluon

$q(k, \sigma) \rightarrow q\left(k^{\prime}, \sigma^{\prime}\right)+g(q)^{*}$,

$g(k, \sigma) \rightarrow g\left(k^{\prime}, \sigma^{\prime}\right)+g(q)^{*}$,

with the four momentum $q=k-k^{\prime} . \sigma$ and $\sigma^{\prime}$ denote the initial and final parton helicities, respectively. The amplitudes for the full process can be expressed as

$\mathcal{M}=J^{\mu}\left(k, k^{\prime} ; \sigma, \sigma^{\prime}\right) \frac{-g_{\mu \nu}}{q^{2}} T^{\nu}$

with

$J^{\mu}\left(k, k^{\prime} ; \sigma, \sigma^{\prime}\right)=g_{s} t^{a} \bar{u}\left(k^{\prime}, \sigma^{\prime}\right) \gamma^{\mu} u(k, \sigma)$

for $q \rightarrow q g$ splitting, and

$$
J^{\mu}\left(k, k^{\prime} ; \sigma, \sigma^{\prime}\right)=g_{s} f^{a b c} \Gamma^{\mu \alpha \beta} \epsilon_{\alpha}^{a}(k, \sigma) \epsilon_{\beta}^{b}\left(k^{\prime}, \sigma^{\prime}\right)^{*}
$$

for $g \rightarrow g g$ splitting. The splitting currents are then expanded by using the polarization vectors with three helicities along the momentum transfer $(q)$ direction:

$$
\begin{aligned}
& J^{\mu}\left(k, k^{\prime} ; \sigma, \sigma^{\prime}\right)=\left(g^{\mu \nu}-\frac{q^{\mu} q^{\nu}}{q^{2}}\right) J_{v}\left(k, k^{\prime} ; \sigma, \sigma^{\prime}\right) \\
& =\sum_{\lambda= \pm, 0}\left(-\eta_{\lambda}\right) \epsilon^{\mu}(q, \lambda) \epsilon^{\nu}(q, \lambda)^{*} J_{v}\left(k, k^{\prime} ; \sigma, \sigma^{\prime}\right) \\
& =\sum_{\lambda= \pm, 0} \mathcal{J}_{\sigma \sigma^{\prime}}^{\lambda}\left(k, k^{\prime}\right) \epsilon^{\mu}(q, \lambda),
\end{aligned}
$$

where the splitting amplitudes [14] are introduced as

$$
\mathcal{J}_{\sigma \sigma^{\prime}}^{\lambda}\left(k, k^{\prime}\right)=-\eta_{\lambda} J_{\mu}\left(k, k^{\prime} ; \sigma, \sigma^{\prime}\right) \epsilon^{\mu}(q, \lambda)^{*} .
$$

The $q \rightarrow q g$ amplitudes are invariant under the boost along the virtual gluon momentum $(q)$ direction, while the $g \rightarrow g g$ ones are not always invariant because of possible gauge choice of the polarization vectors for the external massless gauge bosons. By adopting the common light-cone gauge (10) for all the external and the off-shell gluons, the $g \rightarrow g g$ amplitudes can also be boost invariant along the current momentum direction [14]. By using the boost-invariant splitting amplitudes $\mathcal{J}_{\sigma \sigma^{\prime}}^{\lambda}\left(k, k^{\prime}\right)$ of Ref. [14] and by making the on-shell approximation for the virtual gluon in the hard scattering amplitudes, the azimuthal angle correlations between the initial state splitting $\left(q \rightarrow q g^{*}\right.$ and $\left.g \rightarrow g g^{*}\right)$ plane and the hard scattering plane are reproduced in the collinear limit. For off-shell gluons, however, the longitudinally polarized vector $\epsilon^{\mu}(q, 0)$ in the right-hand side of Eq. (20) behaves badly in the boosted frame, which often leads to severe numerical cancellation among amplitudes in the exact matrix elements.

In the PS gauge, the currents are obtained by the projection:

$$
\begin{aligned}
& J_{\mathrm{PSG}}^{\mu}\left(k, k^{\prime} ; \sigma, \sigma^{\prime}\right)=\tilde{g}^{\mu \nu} J_{\nu}\left(k, k^{\prime} ; \sigma, \sigma^{\prime}\right) \\
& \quad=\sum_{\lambda= \pm, 0}\left(-\eta_{\lambda}\right) \tilde{\epsilon}^{\mu}(q, \lambda) \tilde{\epsilon}^{\nu}(q, \lambda)^{*} J_{\nu}\left(k, k^{\prime} ; \sigma, \sigma^{\prime}\right) \\
& \quad=\sum_{\lambda= \pm, 0} \mathcal{J}_{\sigma \sigma^{\prime}}^{\lambda}\left(k, k^{\prime}\right) \tilde{\epsilon}^{\mu}(q, \lambda) .
\end{aligned}
$$

It is worth noting that the splitting amplitudes (21) are exactly the same between the PS gauge (22) and the Feynman gauge (20) because of current conservation $q_{\mu} J^{\mu}=0$ for the currents of external on-shell quarks (18) and gluons (19). The PSG currents above are, however, free from large longitudinal component even in the highly boosted collinear region, and hence the absolute value squared of the individual Feynman amplitude reproduces the parton-shower behavior in the collinear limit. This shows that the severe cancellation among amplitudes in the original HELAS code that adopts Feynman gauge does not exist in the PS gauge.

The splitting amplitudes for final-state radiations, such as the time-like $\left(q^{2}>0\right) g^{*} \rightarrow q \bar{q}$ and $g^{*} \rightarrow g g$ currents, have the same form as Eq. (22), with $\eta_{\lambda}=1$, in the PS gauge:

$$
J_{\mathrm{PSG}}^{\mu}\left(k, k^{\prime} ; \sigma, \sigma^{\prime}\right)=\sum_{\lambda= \pm, 0} \mathcal{J}_{\sigma \sigma^{\prime}}^{\lambda}\left(k, k^{\prime}\right) \tilde{\epsilon}^{\mu}(q, \lambda)^{*}
$$

with

$\mathcal{J}_{\sigma \sigma^{\prime}}^{\lambda}\left(k, k^{\prime}\right)=-J_{\mu}\left(k, k^{\prime} ; \sigma, \sigma^{\prime}\right) \epsilon^{\mu}(q, \lambda)$

when $q^{2}=\left(k+k^{\prime}\right)^{2}>0$. The boost-invariant splitting amplitudes $\mathcal{J}_{\sigma \sigma^{\prime}}^{\lambda}\left(k, k^{\prime}\right)$ have the same form as obtained in ref. [14], and the off-shell current is free from large components when the virtual gluon is highly boosted.

We note here that the splitting amplitudes (21) and (24) for $g \rightarrow g g$ are not boost invariant for the currents calculated by HELAS codes, JGGXXX [1,2]. This is because the massless gauge boson wave functions in HELAS are fixed by the light-cone gauge with $n^{\mu}=(1,-\mathbf{k} /|\mathbf{k}|)$ by using the three momentum $\mathbf{k}$ of each external photons and gluons. Although this particular choice of the external massless gauge boson wave functions allows HELAS to obtain helicity amplitudes without specifying a particular gauge vector, the splitting amplitudes (21) and (24) evaluated by HELA.S codes do not have the boost-invariant form of Ref. [14], whose absolute 
value squares are the splitting functions of the DGLAP equations. Nevertheless, the HELAS currents have the splitting amplitude form of Ref. [14] in the collinear limit, because the three momenta of external gluons are aligned with the gauge vector (10) of the off-shell gluon. This allows us to express not only the $q \rightarrow q g^{*}$ and $g^{*} \rightarrow q \bar{q}$ but also the $g \rightarrow g g^{*}$ and $g^{*} \rightarrow g g$ currents in terms of the boostinvariant splitting amplitudes of Ref. [14] in the collinear limit.

Summing up, in the PS gauge, the scalar component of the longitudinal state, which behaves badly in the collinear limit, is subtracted systematically, and hence the absolute value squared of each Feynman amplitude reduces to the corresponding parton shower in the collinear limit. The amplitudes are exact even away from the collinear limit, and hence all the interference among different Feynman amplitudes are reproduced exactly, as interference among corresponding partonshower amplitudes.

Before turning to numerical demonstrations in the PS gauge, we briefly mention the special HELAS subroutine JEEXXX, which was introduced to evaluate collinear photon emissions in $e^{+} e^{-}$collisions [1,2]. In order to accurately compute the current with nearly on-shell photon propagator, the original HELAS current JIOXXX is shifted by a term proportional to its four momentum $q^{\mu}$ :

$J_{\mathrm{JEE}}^{\mu}=J_{\mathrm{JIO}}^{\mu}-\frac{J_{\mathrm{JIO}}^{0}}{q^{0}} q^{\mu}$.

When $\left|q^{2}\right| \ll 1$, the $\mu=0$ and 3 components of the current $J_{\text {JIO }}^{\mu}$ grow as $q^{0} / \sqrt{\left|q^{2}\right|}$ because of the longitudinal component. The subtraction (25) suppresses these large components which cause numerical instability because of subtle cancellation among different Feynman amplitudes. In the PS gauge, the current is expressed as

$J_{\mathrm{PSG}}^{\mu}=\tilde{g}^{\mu v} J_{\text {JIOv }}=J_{\mathrm{JIO}}^{\mu}-\frac{n \cdot J_{\mathrm{JIO}}}{n \cdot q} q^{\mu}=J_{\mathrm{JIO}}^{\mu}-\frac{J_{\mathrm{JIO}}^{0}}{|\mathbf{q}|} q^{\mu}$,

which differs from the JEE current only by a term which vanishes in the small $\left|q^{2}\right|$ limit.

\section{Sample results}

In order to demonstrate the power of the PS gauge in numerical calculation of the amplitudes, we study two representative partonic subprocesses:

$u\left(k_{1}\right)+d\left(k_{2}\right) \rightarrow u\left(k_{3}\right)+d\left(k_{4}\right)+g\left(k_{5}\right)$,
$g\left(k_{1}\right)+g\left(k_{2}\right) \rightarrow g\left(k_{2}\right)+g\left(k_{4}\right)+g\left(k_{5}\right)$,

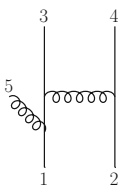

(a)

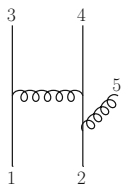

(b)

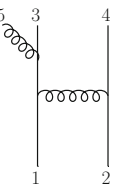

(c)

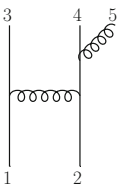

(d)

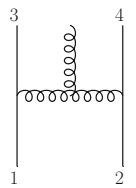

(e)
Fig. 1 Feynman diagrams of the process, $u(1) d(2) \rightarrow u(3) d(4) g(5)$

in the Feynman gauge (with original HELAS) and in the PS gauge with the new HELAS codes presented in Appendix.

For both processes, event samples are generated with head-on collisions of initial partons at $2 \mathrm{TeV}$ centre-of-mass energy with the minimal kinematical cuts for the final-state particles:

$p_{T i}>20 \mathrm{GeV}$,

$\Delta R_{i j}=\sqrt{\left(\eta_{i}-\eta_{j}\right)^{2}+\left(\phi_{i}-\phi_{j}\right)^{2}}>0.4$.

We also introduce the hard-scattering scale as

$\max \left(p_{T i}\right)>200 \mathrm{GeV}$,

which allows us to interpret the amplitudes in the partonshower language, namely as a 2-to-2 hard process of the scale greater than $200 \mathrm{GeV}$ times a parton splitting of the scale down to $k_{T i j}$ of $8 \mathrm{GeV}(=20 \mathrm{GeV} \times 0.4)$. Here, the relative transverse momentum between final-state particles, $k_{T i j}$, is defined as

$k_{T i j}=\min \left(p_{T_{i}}, p_{T_{j}}\right) \Delta R_{i j}$.

Feynman diagrams of the process (27) are shown in Fig. 1. They are categorized into these five types according to gluon radiation topologies; a gluon is radiated from one of the initial-state particles in (a) and (b), from one of the final-state particles in (c) and (d), and from the internal propagator in (e).

In Fig. 2, we show the pseudorapidity of a final-state particle with minimum transverse momentum, $\eta\left(p_{T \text { min }}\right)$, where the result in the PS gauge are plotted in the left-hand side, (A), and that in the Feynman gauge are plotted in the right-hand side, (B). A line with filled circles denotes the differential cross section, i.e. the absolute value squared of the sum of all the amplitudes. On the other hand, lines with triangles (a-d) and open circles (e) show the distribution of the square of each Feynman amplitude depicted in Fig. 1a-e, respectively. A line with filled squares presents the distribution of the sum of the squared amplitudes of each diagram, i.e. without any interference effects among the amplitudes.

The total differential distributions, denoted by lines with filled circles, are identical between the PS gauge (A) and 
Fig. 2 Distributions of pseudorapidity of the final-state particle with minimum transverse momentum for the process $u d \rightarrow u d g$ in the PS gauge (A) and in the Feynman gauge $(\mathbf{B})$. A line with filled circles denotes the total distribution, while lines with triangles (a-d) and open circles (e) show the distribution of the squared amplitude of each type of the Feynman diagrams depicted in Fig. 1. A line with filled squares presents the distribution of the sum of the squared amplitudes of each diagram

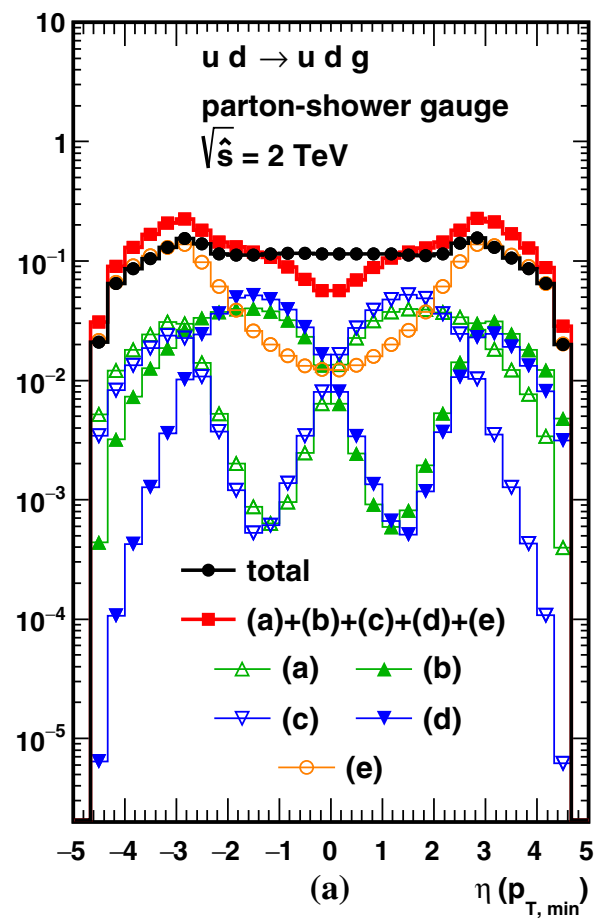

the Feynman gauge (B) as expected. However, the contributions from each amplitude are quite different between the two gauges, especially in the large $\eta$ region. In the Feynman gauge, each squared amplitude in the large $\eta$ region becomes large due to the singularities, causing subtle cancellation of up to two orders of magnitudes among the amplitudes. On the other hand, in the central region $|\eta|<2$, where the contribution from the soft gluon is dominant, all the diagrams contribute almost equally and the contributions from each diagram in the two gauges are very similar. We note that the flat $\eta$ distribution is a consequence of boost invariance of the soft-gluon emission amplitudes.

In order to study the splitting in the initial state, let us focus on the large $\eta$ region in Fig. 2A, where contributions can be explained by the hard-scattering process and the splitting probability in the corresponding parton-shower history. The large positive $\eta$ jet with the smallest $p_{T}$ is mostly $u(3)$ from $u(1) \rightarrow u(3)+g^{*}$ splitting which are common in diagrams (b), (d) and (e). The hard process is then $g^{*}+d(2) \rightarrow d(4)+g(5)$. In the on-shell limit of the virtual gluon $g^{*}$, the sum of the three hard-scattering amplitudes give the physical amplitudes for $g d \rightarrow g d$. The destructive interference observed in the large $\eta$ region of Fig. 2A hence shows the interference among the three amplitudes of the hard-scattering process. In the PS gauge, therefore, one can even observe the interference pattern among hard-scattering amplitudes. Similarly, the large negative $\eta$ jet with the smallest $p_{T}$ is mostly $d(4)$ from $d(2) \rightarrow d(4)+g^{*}$ splitting which are common in diagrams (a), (c) and (e), and the same argument can be applied.
Next, in order to study the final-state splitting, we show the minimum relative transverse momentum between finalstate particles, $k_{T i j \text { min }}$, in Fig. 3. As in Fig. 2, the results in PS gauge are shown in the left panel (A), while those in Feynman gauge are shown in the right (B). Lines with filled circles show the differential cross section obtained by squaring the sum of all the amplitudes, and hence they are identical between (A) and (B). The sum of the square of each diagram is shown by a line with filled squares, which shows subtle cancellation among amplitudes at large $k_{T i j}$ min ( $\gtrsim 100 \mathrm{GeV}$ ) in the Feynman gauge (B).

On the other hand, at small $k_{T i j \min }(\lesssim 30 \mathrm{GeV})$, we can observe the parton-shower behavior, where the hardscattering (with $p_{T}>200 \mathrm{GeV}$ ) process $u d \rightarrow u d$ is followed by collinear gluon emission from final-state $u$ or $d$, corresponding to the Feynman diagrams (c) and (d), respectively, in Fig. 1. Because the contribution of the square of the amplitude (c) and that of (d) are identical, we show their sum by a line with inverted triangles. Likewise the sum of squares of the diagrams (a) and (b) are shown by a line with normal triangles. The dominance of the contribution of the diagrams (c) and (d) at small $k_{T i j \text { min }}$ is observed even in the Feynman gauge (B), because the virtual $u$ and $d$ propagators in (c) and (d) are common between the two gauges.

Now let us turn to the process (28), whose Feynman diagrams are depicted in Fig. 4. Similar to $u d \rightarrow u d g$, the diagrams are categorized into five types. In the type (a), the gluon (5) is emitted from $g(1)$. Likewise $g(5)$ is emitted from $g(2)$ in (b), a virtual gluon splits into $g(3)$ and $g(5)$ in (c), or into $g(4)$ and $g(5)$ in (d). In the diagrams of type (e), $g(5)$ 
Fig. 3 Same as Fig. 2, but for distributions of minimum relative transverse momentum between final-state particles
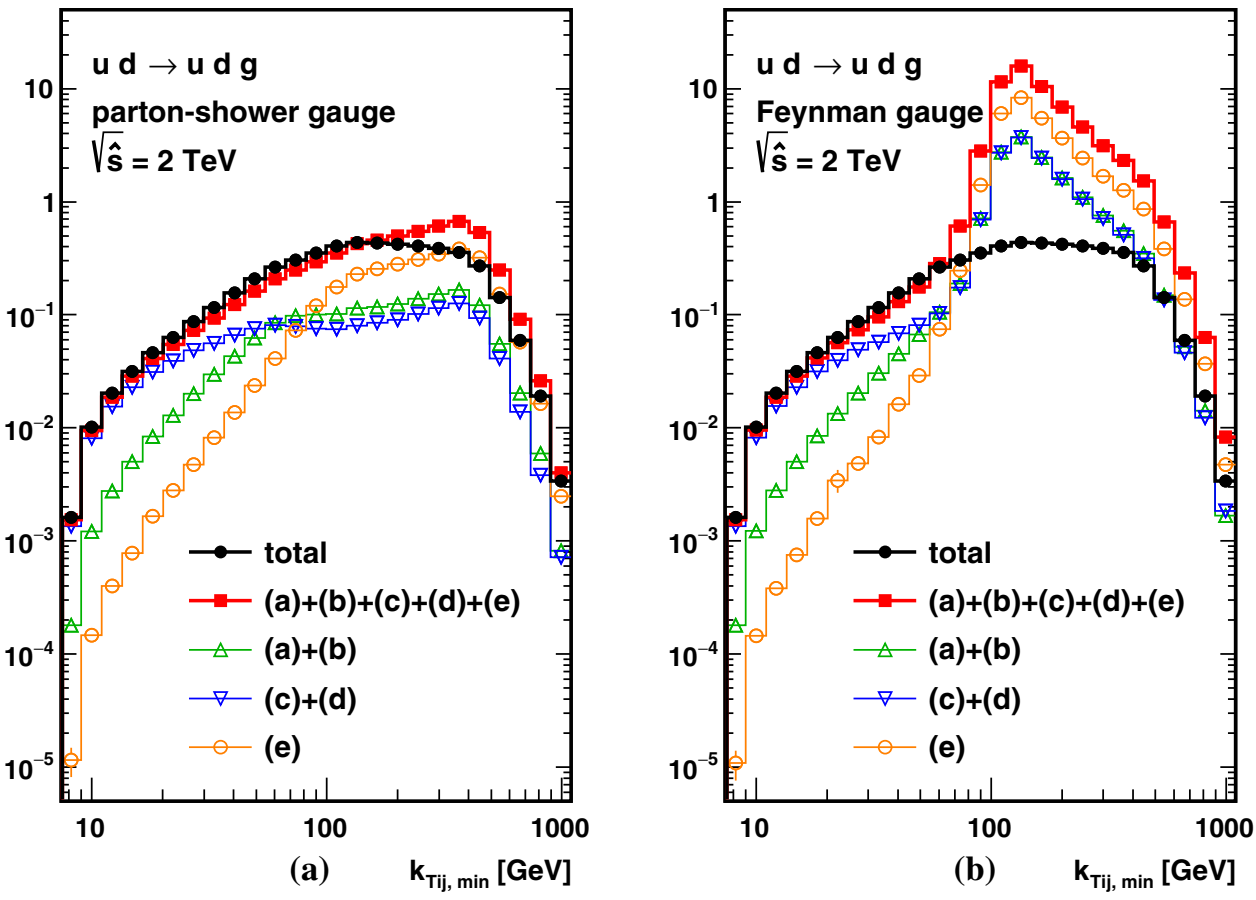

is emitted from an exchanged virtual gluon. For each type, we have $t$-channel (a1)-(e1), $u$-channel (a2)-(e2), as well as the $s$-channel gluon exchange diagrams (a3)-(e3). In addition, although we do not show explicitly in Fig. 4, there are diagrams with the four-point vertex.

Similar to Figs. 2 and 3, we present results for $g g \rightarrow g g g$ in Figs. 5 and 6. The differential cross sections shown by lines with filled circles are given by the absolute value squared of the sum of all the amplitudes including the diagrams with the four-point vertex, and they agree in both gauges as expected. The curves with triangles (open and filled, normal and inverted) and open circles are obtained by squaring the sum of the three diagrams in each column of Fig. 4, such as (a1) + (a2) + (a3) for the type (a), (b1) + (b2) + (b3) for the type (b), etc. We ignore amplitudes with the four-point vertex in these subset of diagrams, since their magnitudes are always found to be small.

In Fig. 5A, the large positive $\eta$ jet comes from the diagrams of type (a), shown by open normal triangles, while the large negative $\eta$ jet comes from the diagrams of type (b), shown by filled normal triangles. In case of (a), the smallest $p_{T}$ jet is $g(5)$, emitted from $g(1)$, and therefore the hard process is $g^{*}+g(2) \rightarrow g(3)+g(4)$. Similarly, for the large negative $\eta$ region, the smallest $p_{T}$ jet is $g(5)$, emitted from $g(2)$, and the hard process is $g(1)+g^{*} \rightarrow g(3)+g(4)$. Even in the central region $|\eta|<2$, where soft gluons with wide angles mostly contribute, the above hard processes are dominant, except at the very central region $|\eta| \sim 0$, where all the diagrams contribute almost equally. In the PS gauge the sum of the absolute value squared of each amplitude can reproduce the physical distribution with small interference effects.

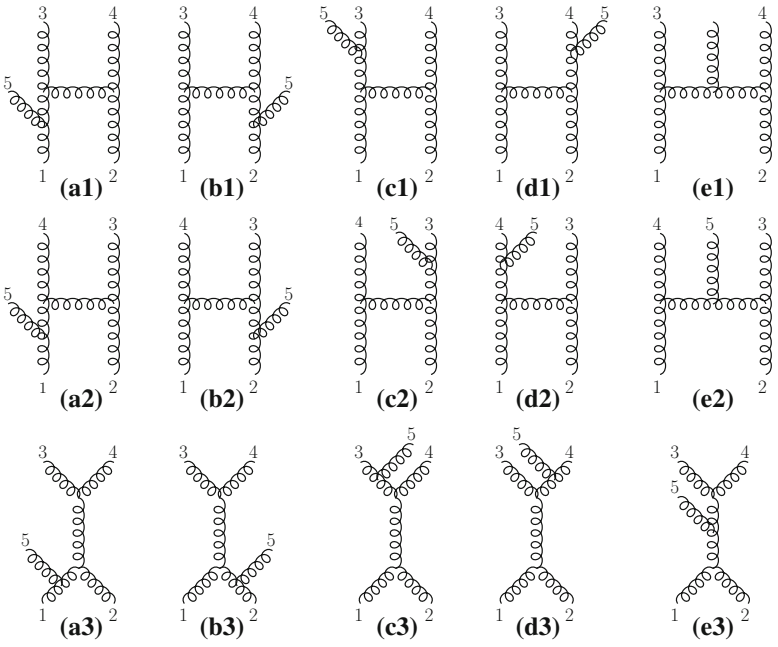

Fig. 4 Feynman diagrams of the process, $g(1) g(2) \rightarrow g(3) g(4) g(5)$

In contrast, in Fig. 5B, all the sub-diagrams have the same behavior; (a) and (b) give the same symmetric $\eta$ distribution, so do (c) and (d). This implies that, in the Feynman gauge, the direction of the virtual gluon three momentum cannot be distinguished and the singular behavior is shared by all the diagrams. The total sum of the squares of the five subamplitudes is given by filled squares, which agrees approximately with the differential cross section in the PS gauge (A), whereas it is one order of magnitude larger than the cross section in the Feynman gauge (B).

In Fig. 6A, similar to the $u d \rightarrow u d g$ case in Fig. 3A, the dominance of the final-state splitting (c)+(d) can clearly be seen in the low $k_{T i j \min }(\lesssim 30 \mathrm{GeV})$ region. This reproduces 
Fig. 5 Distributions of pseudorapidity of the final-state particle with minimum transverse momentum for the process $g g \rightarrow g g g$ in the PS gauge (A) and in the Feynman gauge (B). A line with filled circles denotes the total distribution, while lines with triangles (a-d) and open circles (e) show the distribution of the squared amplitudes of each type of the Feynman diagrams depicted in Fig. 4. A line with distribution of the sum of the squared amplitudes of each diagram filled squares presents the
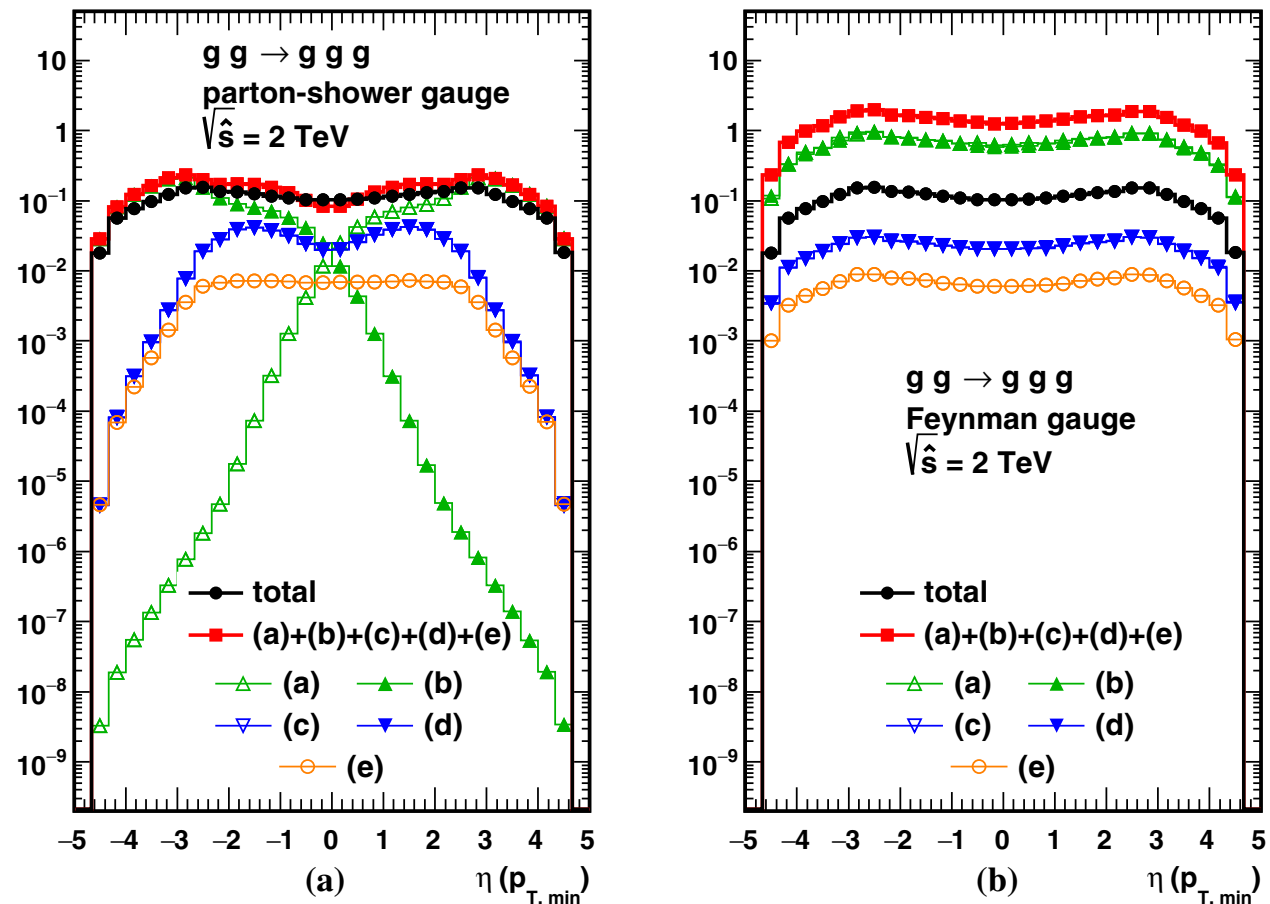

Fig. 6 Same as Fig. 5, but for distributions of minimum relative transverse momentum between final-state particles

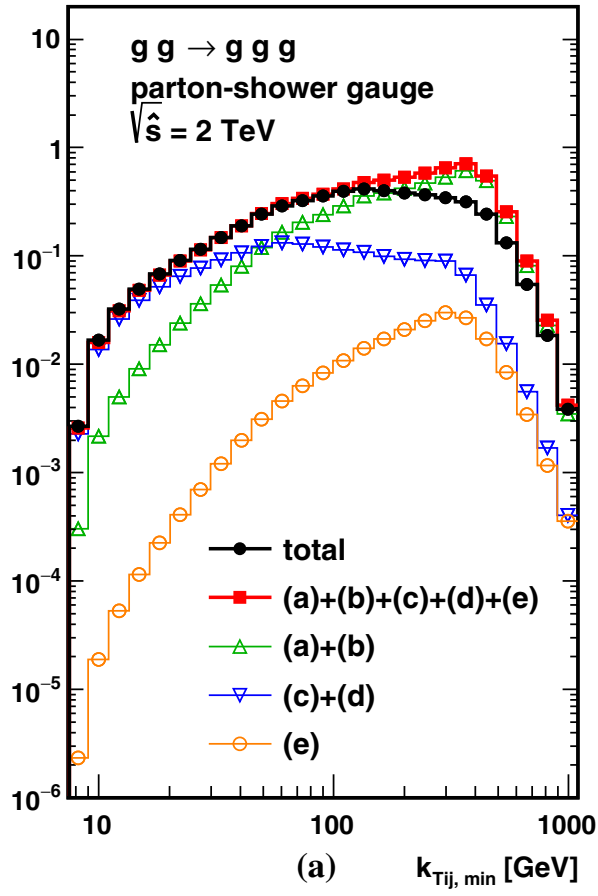

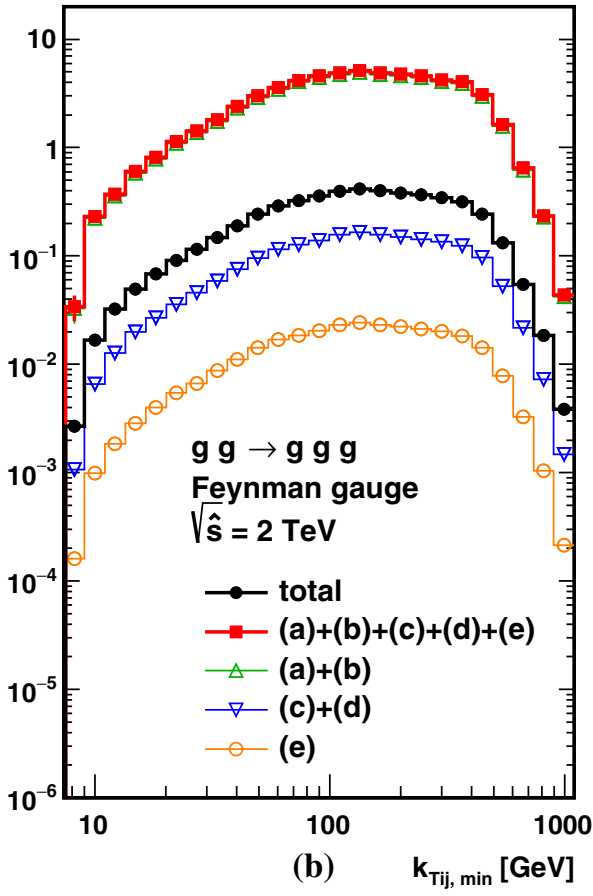

the corresponding parton-shower histories, where the hard scattering gives $g(1)+g(2) \rightarrow g(3)+g(4)$, followed by final-state collinear $g(5)$ emission from $g(3)$ (c) or $g(4)(d)$. In contrast, in the Feynman gauge in Fig. 6B, the contribution from the initial-state splitting (a) + (b) is much larger than that from the final-state splitting $(\mathrm{c})+(\mathrm{d})$, even in the small $k_{T i j \text { min }}$ region. Unlike the final-state splitting $q^{*} \rightarrow q g$, whose collinear-splitting behavior can be observed in both gauges in Fig. 3, the virtual gluon splitting $g^{*} \rightarrow g g$ depends strongly on the gauge because of the large longitudinal component in the collinear limit.

\section{Summary}

We proposed a new propagator form of photons and gluons, in which the scalar component of the longitudinal polarization contribution is subtracted systematically by making use 
of the BRST invariance of the off-shell gauge boson created from physical on-shell states. The propagator has the lightcone gauge form with the gauge vector pointing along the opposite of the off-shell photon or gluon three momentum. We may call the gauge as parton-shower (PS) gauge since in collinear configurations the absolute value squared of each Feynman amplitude reproduces all the singular behaviors of the corresponding parton shower in this gauge. We showed in simple $2 \rightarrow 3$ subprocesses that calculations in the PS gauge can avoid subtle gauge cancellations among Feynman amplitudes that appear in the Feynman gauge. Because of these properties, we believe that amplitudes evaluated in the PS gauge give us deeper insights on scattering processes, and possibly give improved efficiency in event generations, especially with single-diagram-enhanced multi-channel integration [15].

The new HELAS subroutines in the PS gauge are listed in Appendix, and they are publicly available on the web site. ${ }^{4}$ Numerical calculations in the PS gauge can also be done with MadGraph5_aMC@NLO version 2.7.0 or later.

Acknowledgements We would like to thank Olivier Mattelaer for the implementation of the PS gauge into MadGraph5_aMC@NLO. The work of K.M. was supported in part by JSPS KAKENHI Grant No. 18 K03648.

Data Availability Statement This manuscript has no associated data or the data will not be deposited. [Authors' comment: There are no external data associated with the manuscript.]

Open Access This article is licensed under a Creative Commons Attribution 4.0 International License, which permits use, sharing, adaptation, distribution and reproduction in any medium or format, as long as you give appropriate credit to the original author(s) and the source, provide a link to the Creative Commons licence, and indicate if changes were made. The images or other third party material in this article are included in the article's Creative Commons licence, unless indicated otherwise in a credit line to the material. If material is not included in the article's Creative Commons licence and your intended use is not permitted by statutory regulation or exceeds the permitted use, you will need to obtain permission directly from the copyright holder. To view a copy of this licence, visit http://creativecomm ons.org/licenses/by/4.0/.

Funded by SCOAP ${ }^{3}$.

\section{Appendix A: Sample codes in the parton-shower gauge}

In this section we list HELAS subroutines that are needed to compute off-shell vector currents in the PS gauge. For future extension of HELAS we modify the internal structure of HELAS wave functions. In the original HELAS wave functions the four momentum of particles is stored in the last two components of the complex six-dimensional array. In new HELAS which includes new PS-gauge calculation

\footnotetext{
${ }^{4}$ HELAS/MadGraph/MadEvent Home Page: https://madgraph.ipmu. jp/IPMU/.
}

the first two components of the wave functions contain the four momentum ${ }^{5}$ because the extension of the array of wave functions becomes possible to include further information. We note that the original HELAS library was coded by For$\operatorname{tran} 77$, while the new library is coded by Fortran 90.

\section{A.1 Parameter file and utility subroutine}

New parameters to control helicity amplitude calculation are included in the new HELAS library. In helas_param. 190 (List 1) a new flag, helas_mode, is introduced. Feynman gauge is used when helas_mode $=1$ and PS gauge is used when helas_mode $=2$ for massless gauge boson propagators. This flag can be changed by calling hlmode (helas_mode) (List 2), then all the gauge definition for massless gauge-boson propagators is changed.

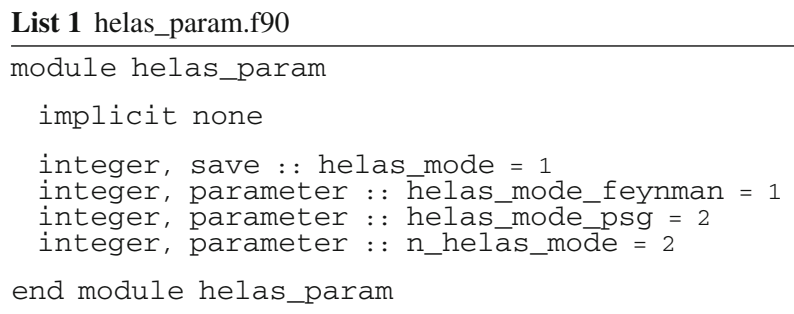

List 2 hlmode.f90

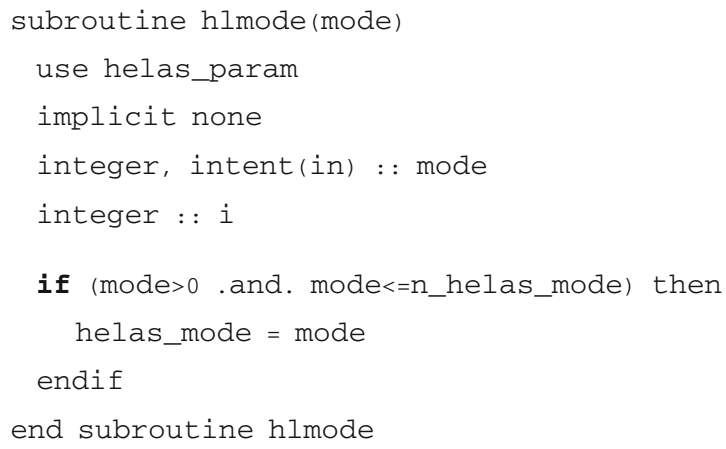

\section{A.2 Off-shell vector-current subroutines}

We introduce subroutines to compute off-shell massless vector-boson currents in the PS gauge that are used in this paper. The list of the arguments in these subroutines is the same as that in the original HELAS library [2], so that it is not necessary to modify amplitude computation program to use PS gauge instead of Feynman gauge.

\section{A.2.1 jioxxx}

The function, jioxxx (List 3), computes an off-shell vector current attached to an external fermion line. The vector-boson

\footnotetext{
5 ALOHA [5] employs this structure.
} 
propagator is given in the PS gauge for a massless vector if the flag, helas_mode, is set to 2 .

List 3 jioxxx.f90 subroutine jioxxx(fi,fo,gc, vmass, vwidth, jio) use helas_param

implicit none

double complex, intent(in) : : fi(6), fo(6)

double complex, intent(in) : : gc(2)

double precision, intent(in) : : vmass, vwidth

double complex, intent(out) : : jio(6)

double complex : : jj $(0: 4)$

double complex :: j12(0:4)

double complex : : js, d

double complex : : js1,js2

double complex :: $\mathrm{q}(0: 4)$

double precision :: qabs2, qabs

double precision : : q2, vm2

double complex : : cm2

double precision : : $\mathrm{n}(0: 4)$

double precision : : nq

double complex, parameter : $\mathrm{ci}=(0 . \mathrm{d} 0,1 . \mathrm{d} 0)$

jio $(1: 2)=f o(1: 2)-f i(1: 2)$

$\mathrm{q}(0)=-\mathrm{dble}(\mathrm{jio}(1))$

$q(1)=-\operatorname{dble}(j i o(2))$

$q(2)=-\operatorname{dimag}(j i \circ(2))$

$\mathrm{q}(3)=-\operatorname{dimag}(j i o(1))$

$\mathrm{q}(4)=-\mathrm{Ci} * \operatorname{vmass}$

qabs $2=\operatorname{dble}(\mathrm{q}(1)) * * 2+\mathrm{dble}(\mathrm{q}(2)) * * 2+\mathrm{dble}(\mathrm{q}(3)) * * 2$

qabs $=\operatorname{sqrt}($ qabs 2$)$

$\mathrm{q} 2=\mathrm{db} l \mathrm{e}(\mathrm{q}(0)) * * 2-q a b s 2$

$\mathrm{vm} 2=$ vmass $* * 2$

$j 12(0)=g C(1) *(f O(5) * f i(3)+f o(6) * f i(4)) \&$ $+g C(2) *(f o(3) * f i(5)+f o(4) * f i(6))$

$j 12(1)=-g C(1) *(f \circ(5) * f i(4)+f o(6) * f i(3)) \&$ $+g C(2) *($ fo $(3) * f i(6)+f o(4) * f i(5))$

$j 12(2)=(\operatorname{gc}(1) *($ fo( 5$) * f i(4)-f o(6) * f i(3)) \&$ $+g C(2) *(-f o(3) * f i(6)+f o(4) * f i(5))) * C i$

$j 12(3)=g C(1) *(-f o(5) * f i(3)+f o(6) * f i(4)) \&$ $+g C(2) *($ fo $(3) * f i(5)-f o(4) * f i(6))$

$\mathrm{d}=1 . \mathrm{d} 0 / \mathrm{dcmp} l \mathrm{x}\left(\mathrm{q} 2-\mathrm{vm} 2, \mathrm{vmass}{ }^{*} \mathrm{vwidth}\right)$

if $(\mathrm{vmass} /=0 . \mathrm{d} 0)$ then

$\mathrm{cm} 2=$ dcmplx $(\mathrm{vm} 2,-$ vmass $*$ vwidth $)$

$j s=(q(0) * j 12(0)-q(1) * j 12(1)-\&$ $q(2) * j 12(2)-q(3) * j 12(3)), \quad c m 2$

$j j(0: 3)=j 12(0: 3)-q(0: 3) * j s$

else

$j j(0: 3)=j 12(0: 3)$

end if

jio $(3: 6)=j j(0: 3) * d$

if (helas_mode==helas_mode_psg) then

if (vmass $==0 . d 0$ ) then

if (qabs $2>=\operatorname{dble}(\mathrm{q}(0)) * * 2)$ then

$\mathrm{n}(0)=\operatorname{sign}(1 \cdot \mathrm{d} 0, \mathrm{dble}(\mathrm{q}(0)))$

$\mathrm{n}(1)=-\mathrm{dble}(\mathrm{q}(1)) / \mathrm{qabs}$

$n(2)=-d b l e(q(2)) /$ qabs

$n(3)=-d b l e(q(3)) / q a b s$

else

$n(0)=1 . d 0$

$\mathrm{n}(1)=0 . \mathrm{d} 0$

$\mathrm{n}(2)=0 . \mathrm{d} 0$

$n(3)=-1 . d 0$

endif

$\mathrm{nq}=\mathrm{n}(0) * \mathrm{dble}(\mathrm{q}(0))-\mathrm{n}(1) * \mathrm{dble}(\mathrm{q}(1)) \quad \&$ $-\mathrm{n}(2) * \mathrm{able}(\mathrm{q}(2))-\mathrm{n}(3) * \mathrm{able}(\mathrm{q}(3))$

$\begin{aligned} j s 1 & =(n(0) * j 12(0)-n(1) * j 12(1) \& \\ & -n(2) * j 12(2)-n(3) * j 12(3)) / n q\end{aligned}$

$j s 2=(q(0) * j 12(0)-q(1) * j 12(1) \&$

$-q(2) * j 12(2)-q(3) * j 12(3)) / n q$

jio $(3: 6)=(j 12(0: 3)-q(0: 3) * j \sin -n(0: 3) * j s 2) *$ a

endif

end if

end subroutine jioxxx

\section{A.2.2 jggxxx}

The function, jggxxx (List 4), computes an off-shell gluon current from the three-point gluon vertex attached with the gluon propagator depending on the helas_mode flag.

List 4 jggxxx.f90

subroutine jggxxx(v1,v2,g, jgg)

use helas_param

implicit none

double complex, intent(in) : : v1(6), v2 (6)

double precision, intent(in) : : $g$

double complex, intent(out) : : jgg(6)

double complex : : j12(0:3), v12

double precision :: $\mathrm{p} 1(0: 3), \mathrm{p} 2(0: 3), \mathrm{q}(0: 3), \mathrm{q} 2$

double precision : : d

double complex :: p23v1, p31v2

double complex : : js1, js2

double precision : : qabs, qabs2

double precision : : nq

double precision : : $\mathrm{n}(0: 3)$

$j g g(1: 2)=\mathrm{v} 1(1: 2)+\mathrm{v} 2(1: 2)$

$\mathrm{p} 1(0)=\mathrm{dble}(\mathrm{v} 1(1))$

$\mathrm{p} 1(1)=\mathrm{dble}(\mathrm{V} 1(2))$

$\mathrm{p} 1(2)=\operatorname{dimag}(\mathrm{V} 1(2))$

$\mathrm{p} 1(3)=\operatorname{dimag}(\mathrm{v} 1(1))$

$\mathrm{p} 2(0)=\mathrm{dble}(\mathrm{v} 2(1))$

$\mathrm{p} 2(1)=$ dble $(\mathrm{V} 2(2))$

$\mathrm{p} 2(2)=\operatorname{dimag}(\mathrm{v} 2(2))$

$\mathrm{p} 2(3)=\operatorname{dimag}(\mathrm{v} 2(1))$

$\mathrm{q}(0)=-(\mathrm{p} 1(0)+\mathrm{p} 2(0))$

$\mathrm{q}(1)=-(\mathrm{p} 1(1)+\mathrm{p} 2(1))$

$q(2)=-(\mathrm{p} 1(2)+\mathrm{p} 2(2))$

$\mathrm{q}(3)=-(\mathrm{p} 1(3)+\mathrm{p} 2(3))$

qabs $2=q(1) * * 2+q(2) * * 2+q(3) * * 2$

qabs $=$ sqrt $($ qabs 2$)$

$\mathrm{q} 2=\mathrm{dble}(\mathrm{q}(0)) * * 2-q a b s 2$

$\mathrm{V} 12=\mathrm{V} 1(3) * \mathrm{~V} 2(3)-\mathrm{V} 1(4) * \mathrm{~V} 2(4)-\mathrm{V} 1(5) * \mathrm{~V} 2(5)-\mathrm{V} 1(6) * \mathrm{~V} 2$ (6)

$\mathrm{p} 23 \mathrm{v} 1=(\mathrm{p} 2(0)-\mathrm{q}(0)) * \mathrm{v} 1(3)-(\mathrm{p} 2(1)-\mathrm{q}(1)) * \mathrm{v} 1(4) \&$ - $(\mathrm{p} 2(2)-\mathrm{q}(2)) * \mathrm{v} 1(5)-(\mathrm{p} 2(3)-\mathrm{q}(3)) * \mathrm{v} 1(6)$

$\mathrm{p} 31 \mathrm{v} 2=(\mathrm{q}(0)-\mathrm{p} 1(0)) * \mathrm{v} 2(3)-(\mathrm{q}(1)-\mathrm{p} 1(1)) * \mathrm{v} 2(4) \&$ $-(\mathrm{q}(2)-\mathrm{p} 1(2)) * \mathrm{v} 2(5)-(\mathrm{q}(3)-\mathrm{p} 1(3)) * \mathrm{v} 2(6)$

$\mathrm{j} 12(0: 3)=-\mathrm{g} *((\mathrm{p} 1(0: 3)-\mathrm{p} 2(0: 3)) * \mathrm{v} 12+\mathrm{p} 23 \mathrm{v} 1 * \mathrm{v} 2$ $(3: 6)+\&$ $\mathrm{p} 31 \mathrm{v} 2 * \mathrm{v} 1(3: 6))$

$\mathrm{d}=1 . \mathrm{d} 0 / \mathrm{q} 2$

$j g g(3: 6)=j 12(0: 3) * d$

if (helas_mode==helas_mode_psg) then 


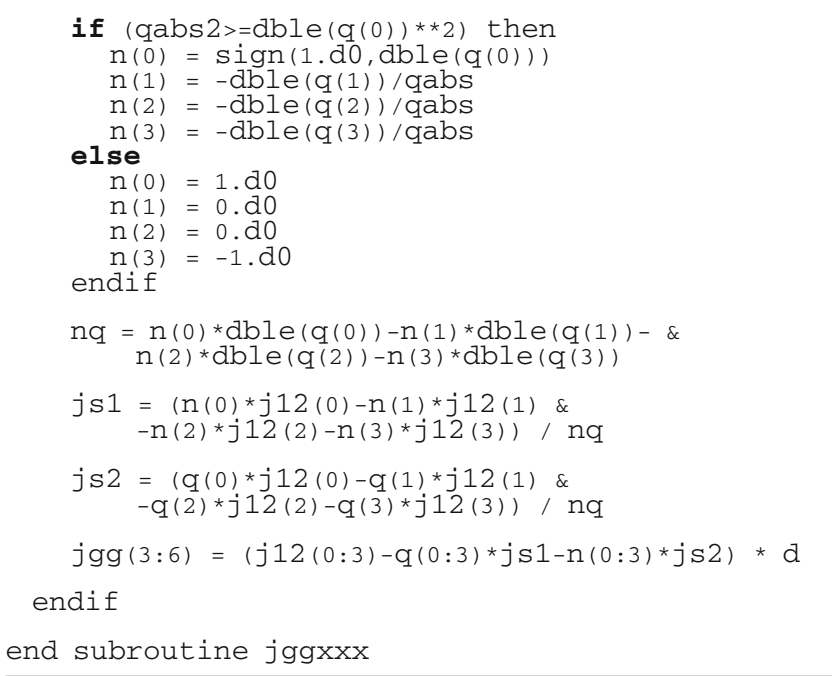

\section{A.2.3 jgggxx}

The function, jgggxx (List 5), computes an off-shell gluon current from the fourt-point gluon vertex attached with the gluon propagator depending on the helas_mode flag.

List 5 jgggxx.f90

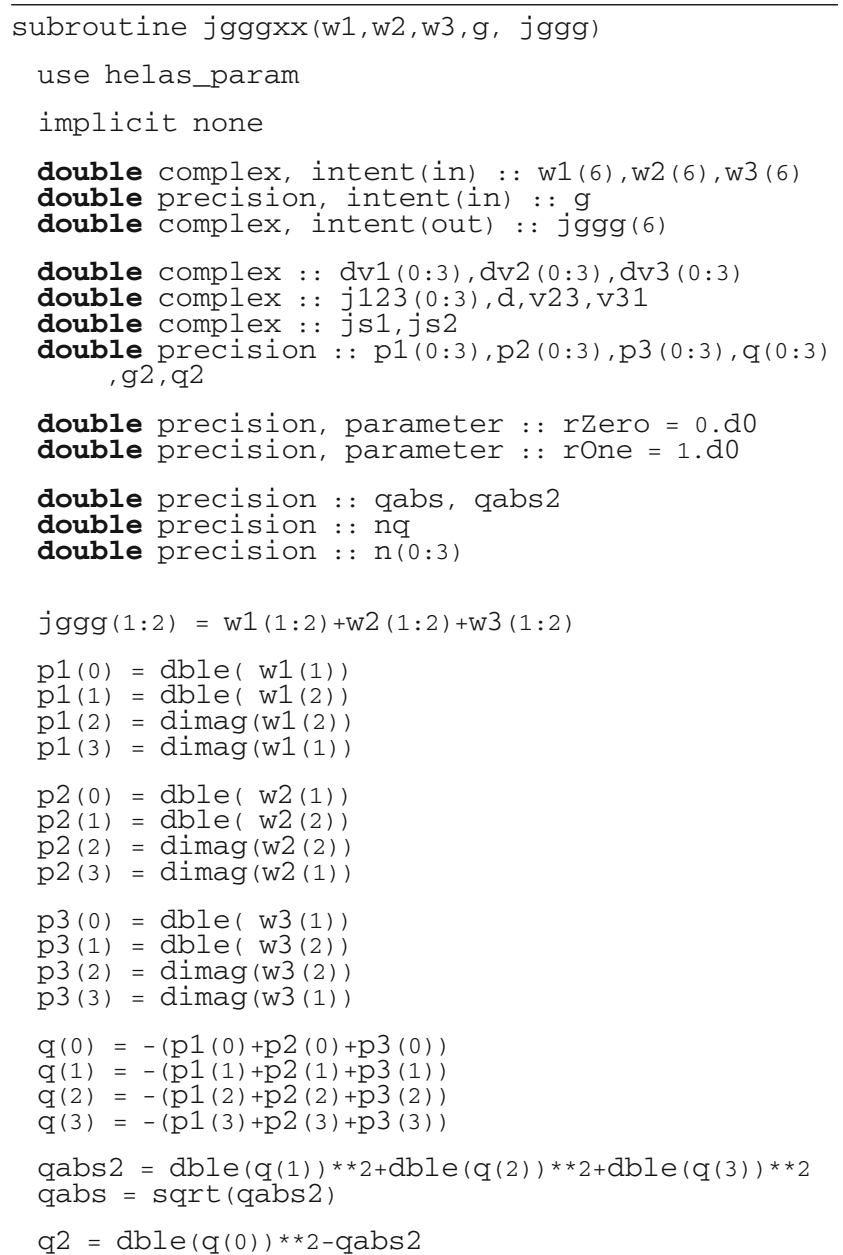

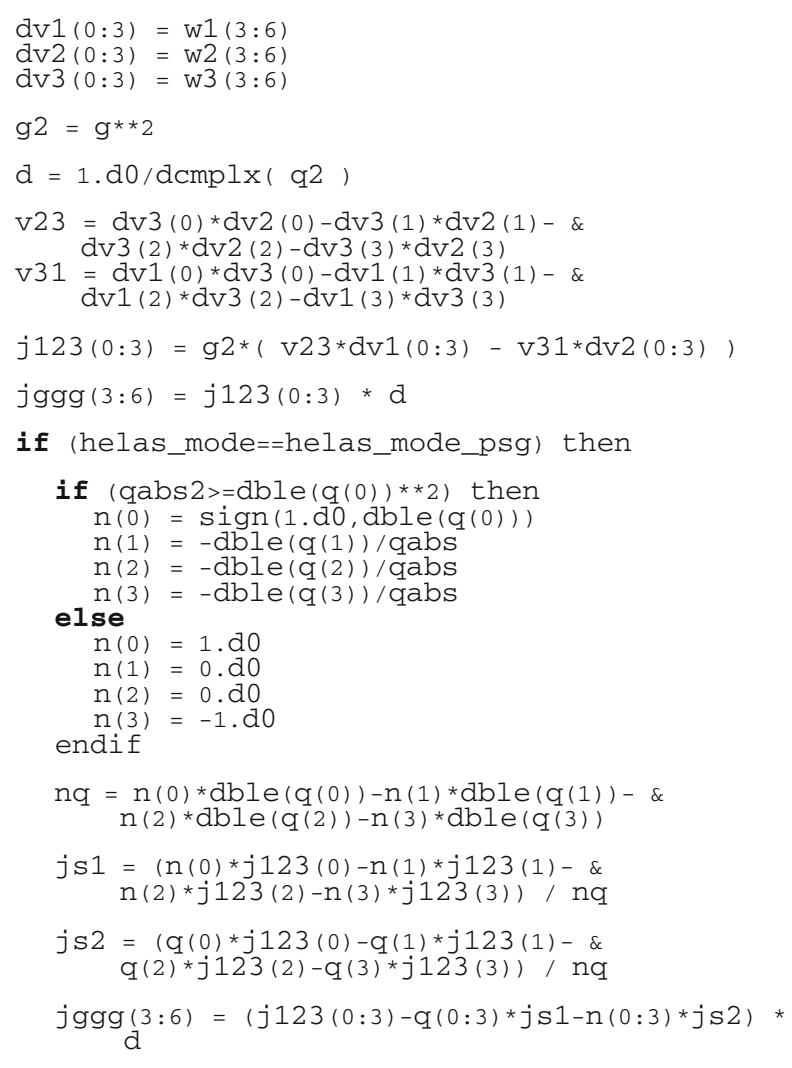

endif

end subroutine jgggxx

\section{References}

1. K. Hagiwara, H. Murayama, I. Watanabe, Search for the Yukawa interaction in the process $e^{+} e^{-} \rightarrow t \bar{t} Z$ at TeV linear colliders. Nucl. Phys. B 367, 257-286 (1991). https://doi.org/10.1016/ 0550-3213(91)90017-R

2. H. Murayama, I. Watanabe, K. Hagiwara, HELAS: HELicity amplitude subroutines for Feynman diagram evaluations, KEK91-11

3. J. Alwall, R. Frederix, S. Frixione, V. Hirschi, F. Maltoni, O. Mattelaer et al., The automated computation of tree-level and nextto-leading order differential cross sections, and their matching to parton shower simulations. JHEP 07, 079 (2014). https://doi.org/ 10.1007/JHEP07(2014)079. arXiv:1405.0301

4. J. Alwall, P. Demin, S. de Visscher, R. Frederix, M. Herquet, F. Maltoni et al., MadGraph/MadEvent v4: the new web generation. JHEP 09, 028 (2007). https://doi.org/10.1088/1126-6708/2007/09/028. arXiv:0706.2334

5. P. de Aquino, W. Link, F. Maltoni, O. Mattelaer, T. Stelzer, ALOHA: automatic libraries of helicity amplitudes for feynman diagram computations. Comput. Phys. Commun. 183, 2254-2263 (2012). https://doi.org/10.1016/j.cpc.2012.05. 004. arXiv: 1108.2041

6. C. Degrande, C. Duhr, B. Fuks, D. Grellscheid, O. Mattelaer, T. Reiter, UFO-the universal FeynRules output. Comput. Phys. Commun. 183, 1201-1214 (2012). https://doi.org/10.1016/j.cpc. 2012.01.022. arXiv: 1108.2040 
7. K. Hagiwara, H. Iwasaki, A. Miyamoto, H. Murayama, D. Zeppenfeld, Single weak boson production at $\mathrm{TeV} e^{+} e^{-}$colliders. Nucl. Phys. B 365, 544-596 (1991). https://doi.org/10.1016/ 0550-3213(91)90442-Z

8. Z. Nagy, D.E. Soper, Parton showers with quantum interference. JHEP 09, 114 (2007). https://doi.org/10.1088/1126-6708/2007/ 09/114. arXiv:0706.0017

9. Z. Nagy, D.E. Soper, A parton shower based on factorization of the quantum density matrix. JHEP 06, 097 (2014). https://doi.org/10. 1007/JHEP06(2014)097. arXiv:1401.6364

10. C. Becchi, A. Rouet, R. Stora, Renormalization of gauge theories. Ann. Phys. 98, 287-321 (1976). https://doi.org/10.1016/ 0003-4916(76)90156-1

11. I.V. Tyutin, Gauge invariance in field theory and statistical physics in operator formalism, LEBEDEV-75-39
12. J. Chen, T. Han, B. Tweedie, Electroweak splitting functions and high energy showering. JHEP 11, 093 (2017). https://doi.org/10. 1007/JHEP11(2017)093. arXiv:1611.00788

13. J. Chen, K. Hagiwara, J. Kanzaki, in preparation

14. K. Hagiwara, Q. Li, K. Mawatari, Jet angular correlation in vector-boson fusion processes at hadron colliders. JHEP 07, 101 (2009). https://doi.org/10.1088/1126-6708/2009/07/101. arXiv:0905.4314

15. F. Maltoni, T. Stelzer, MadEvent: automatic event generation with MadGraph. JHEP 02, 027 (2003). https://doi.org/10.1088/ 1126-6708/2003/02/027. arXiv:hep-ph/0208156 